\title{
Aspectos psicológicos e reabilitação profissional: revisão de literatura
}

\author{
Psychological aspects and vocational \\ rehabilitation: A literature review
}

\author{
Patricia CANAL ${ }^{1}$ \\ Roberto Moraes CRUZ
}

\begin{abstract}
Resumo
As doenças ocupacionais podem gerar prejuízos para a saúde mental dos trabalhadores, o que resulta em incapacidade laboral. A partir dos programas de reabilitação profissional, esses trabalhadores podem voltar a exercer uma atividade laborativa. Este artigo tem como objetivo realizar um levantamento bibliográfico a respeito da reabilitação profissional e dos aspectos psicológicos relacionados. Realizou-se rastreamento nas bases de dados nacionais e internacionais, o que resultou em um total de 70 artigos, posteriormente organizados nas categorias conceitos e políticas de reabilitação profissional, aspectos psicológicos em reabilitação profissional e processos de intervenção em reabilitação profissional. Estudos no campo da reabilitação profissional envolvendo aspectos da saúde mental são recentes, e observou-se um aumento nas publicações a partir da década de 2000. Aponta-se a necessidade da realização de estudos nessa área, a fim de ampliar a produção sobre os aspectos da saúde mental em reabilitação profissional.
\end{abstract}

Unitermos: Aspectos psicológicos; Incapacidade; Reabilitação profissional; Saúde mental.

\begin{abstract}
Occupational diseases may cause damage to workers' mental health resulting in the incapacity to work. Through vocational rehabilitation programs these workers can be helped to return to work. This article is a literature review of studies related to vocational rehabilitation of workers suffering from psychological damage. Data were collected from national and international databases, resulting in a total of 70 articles that were organized into the following categories: concepts and policies of vocational rehabilitation, psychological aspects of vocational rehabilitation, and interventions in vocational rehabilitation. Studies in the field of vocational rehabilitation that involve aspects of mental health are recent, with an increase in publications since the 2000s. New studies within this field of knowledge are needed, aiming to increase the production related to mental health aspects in vocational rehabilitation.
\end{abstract}

Uniterms: Psychological aspects; Inability; Rehabilitation vocational; Mental health.

Conforme a Organização Internacional do Trabalho (OIT), os programas de reabilitação profissional têm como objetivo permitir que o sujeito com incapaci- dade obtenha, conserve um emprego e progrida nele, para que se promova assim a integração ou a reintegração dessa pessoa à sociedade. A Reabillitação

$\boldsymbol{\nabla} \mathbf{v} \boldsymbol{v}$

1 Universidade Federal de Santa Catarina, Centro de Filosofia e Ciências Humanas, Programa de Pós-Graduação em Psicologia. Campus Universitário Reitor João David Ferreira Lima, Trindade, 88040-970, Florianópolis, SC, Brasil. Correspondência para/Correspondence to: P. CANAL. E-mail: <pcanal@unochapeco.edu.br>. 
Profissional compreende todas as ações sistemáticas e organizadas para dar apoio ao trabalhador incapacitado e promover sua reintegração ao emprego (Watzke \& Galvao, 2008). Ao trabalhar em reabilitação, o profissional faz uso de processos interventivos que visam restituir as capacidades do indivíduo e reintegrá-lo de forma completa em todas as áreas de sua vida.

Os Transtornos Mentais e do Comportamento (TMC) estão geralmente entre os diagnósticos mais frequentes atribuídos aos trabalhadores afastados do emprego por incapacidade (Brasil, 2008). O sofrimento do trabalhador pode decorrer também da própria situação de afastamento e da condição de incapacidade (Ramos, Tittoni \& Nardi, 2008). A intervenção em reabilitação profissional surge como dispositivo importante para promover a reinserção social dos trabalhadores adoecidos.

Neste artigo, aborda-se o conceito de reabilitação profissional e descrevem-se os aspectos psicológicos envolvidos nos processo de reabilitação profissional e a necessidade da inclusão de estratégias de intervenção psicológica nos programas de reabilitação profissional para garantir sua efetividade.

\section{Método}

Este estudo objetivou realizar um levantamento bibliográfico a respeito da reabilitação profissional e seus aspectos psicológicos. Foi realizado rastreamento nas bases de dados nacionais e internacionais Psycarticles, Science Direct, SciELO, Springer Journals e PubMed.

O levantamento de dados ocorreu no período de julho de 2009 a julho de 2010, e os descritores utilizados na busca foram: reabilitação profissional, transtornos mentais, trabalho, retorno ao trabalho e incapacidade. Os respectivos termos em inglês e espanhol foram utilizados para a busca em periódicos internacionais. Foram incluídos os artigos que atendiam os objetivos da pesquisa.

Levantou-se um total de 70 artigos, posteriormente organizados em três categorias teóricas: conceitos e políticas de reabilitação profissional, aspectos psicológicos em reabilitação profissional e processos de intervenção em reabilitação profissional.
Sobre a intervenção em reabilitação profissional, foram mapeadas as experiências descritas na literatura científica. Como critério de inclusão, adotou-se a intervenção psicológica em casos de afastamento do trabalho incluídas ou não dentro de um programa estruturado ou nomeado de reabilitação profissional. Foram identificadas 7 descrições de experiência em intervenção psicológica (Bartilotti, Andrade, Varandas, Ferreira \& Cabral, 2009; Hatinen, Kinnunen, Pekkonen \& Kalimo, 2007; Lima et al., 2010; Marques, Queirós \& Rocha, 2006): 5 no Brasil e um na Finlândia.

\section{Reabilitação profissional: conceitos e políticas}

A palavra reabilitar deriva do latim habilitare, que significa tornar hábil ou apto, preparar, prover do necessário, adquirir habilitações. Reabilitar significa então adquirir novamente uma habilidade perdida ou reduzida. Tradicionalmente, o conceito de reabilitação tem sido apresentado pelos autores da área como um processo de restaurar uma capacidade perdida ou reduzida. Em consonância à origem etimológica do termo, Reagles, Wright e Butler (1971) atribuem ao processo de reabilitação o significado de restauração, ou seja, adquirir ou readquirir algo que foi perdido, objetivando a mudança ou melhora do indivíduo nos aspectos físico, mental, social e cultural.

Matsuo (2002) refere que a reabilitação tem sido tradicionalmente estudada a partir de uma abordagem biomédica, consequentemente a atenção tem sido realizada com foco nos aspectos clínicos do tratamento médico e na recuperação da capacidade física do indivíduo a ser reabilitado. A autora considera importante que se vá além dessa perspectiva e se busque uma compreensão dos mecanismos existentes no processo de reabilitação. Lollar (2008) cita a definição proposta pelo Dicionário Médico de Stedman, que caracteriza a reabilitação como restauração - seguinte a uma doença, enfermidade ou trauma -, de uma habilidade para a funcionalidade de modo normal ou próximo ao normal. O autor chama atenção para a noção, implícita neste conceito, de reabilitação como um processo focado no indivíduo, e propõe que se estude a área de forma mais ampla a partir das noções de incapacidade e saúde preconizadas pela Organização Mundial de Saúde (OMS) em seu sistema de classificação de incapacidades (OMS, 2004). 
A incapacidade está estreitamente relacionada à área de reabilitação, e é entendida como uma dificuldade para realizar determinados tipos de funções. A Classificação Internacional de Funcionalidade, Incapacidade e Saúde (OMS, 2004) aponta dois modelos de entendimento da incapacidade e baseia-se na integração desses dois modelos: o modelo biomédico, que considera a doença como um problema do indivíduo, tendo causa direta a doença, trauma ou problema de saúde, e requer intervenção individual na forma de tratamento de saúde; e o modelo social, que considera a incapacidade um problema social, e uma questão de integração plena do indivíduo na sociedade. Partindo-se desse pressuposto, a incapacidade deixa de ser um atributo de um indivíduo e se torna um conjunto complexo de condições, muitas das quais criadas pelo ambiente social. A responsabilidade de fazer as modificações ambientais necessárias para a participação plena das pessoas com incapacidades em todas as áreas da vida social é entendida como coletiva e da sociedade.

Momm e Geiecker (1998) discutem o conceito de incapacidade e apontam para a dificuldade de se chegar a um acordo no entendimento do termo. Segundo os autores, o significado do termo incapacidade em diferentes línguas remete à ideia de menor valor, menor capacidade, desvio, privação, limitação, o que implica o entendimento do termo como um problema exclusivo do indivíduo. No entanto, em consonância com o modelo social de incapacidade, os autores citados afirmam que o efeito da suposta disfunção tem relação com o ambiente, o que torna a incapacidade um conceito social e não exclusivamente um atributo do indivíduo.

O modelo social de incapacidade também é proposto por Pledger (2003) como alternativa ao modelo médico. O chamado novo modelo de incapacidade tem foco na funcionalidade dentro de um contexto socioecológico, e reforça a ideia de que os fatores externos contribuem para a experiência de incapacidade, que é produto da interação de características individuais e características do ambiente social. Os modelos de incapacidade norteiam as políticas voltadas para a área e são fundamentais para a definição dos critérios de elegibilidade dos sujeitos da reabilitação, disponibilidade dos serviços e acesso aos recursos. Da mesma forma, o modo como os profissionais concebem a incapacidade pode ser determinante para embasar a intervenção.

Entre os objetivos da reabilitação profissional está a recolocação no mercado de trabalho de trabalhadores parcialmente incapacitados para o exercício laboral devido a um acidente de trabalho ou doença ocupacional. A Organização Internacional do Trabalho (OIT), na Convenção no 159, preconiza como finalidade da reabilitação profissional "permitir que a pessoa deficiente obtenha e conserve um emprego e progrida no mesmo, e que se promova, assim, a integração ou a reintegração dessa pessoa na sociedade" (ILO, 1993).

No Brasil, as diretrizes para a reabilitação profissional são estabelecidas no âmbito da Previdência Social. O Decreto 3.048/99 a define como a assistência (re)educativa e de (re)adaptação profissional, instituída sob a denominação genérica de habilitação e reabilitação profissional, visa proporcionar aos beneficiários incapacitados, parcial ou totalmente para o trabalho, em caráter obrigatório, independentemente de carência, e às pessoas portadores de deficiência, os meios indicados para proporcionar o reingresso no mercado de trabalho e no contexto em que vivem (Brasil, 1999).

Dessa forma, as ações em reabilitação profissional acontecem por meio de: avaliação do potencial laborativo, orientação e acompanhamento da programação profissional, articulação com a comunidade e acompanhamento e pesquisa da fixação no mercado de trabalho. Observa-se que essa diretriz não contempla o tratamento e a intervenção em reabilitação, o que chama a atenção para a necessidade de se pensar em modelos de intervenção ainda não consolidados na políticas públicas do país.

\section{Abordagens em reabilitação profissional}

Atualmente, entre os desafios que enfrentam os profissionais envolvidos com intervenções em reabilitação profissional, está a contradição entre a necessidade de colocação de pessoas com incapacidade e as demandas das organizações empregadoras. O mercado de trabalho requer qualificações que não conseguem ser alcançadas às vezes nem pela população em geral, e em menor grau ainda pelas pessoas consideradas incapazes, principalmente portadoras de algum tipo de sofrimento psíquico (Watzke \& Galvao, 2008). É neces- 
sário então que se pense em modelos de reabilitação profissional que diminuam essa contradição, reduzam a incapacidade dos sujeitos e atuem sobre as condições ambientais para que as exigências do mercado se aproximem mais das habilidades desses sujeitos.

Simonelli, Camarotto, Bravo e Vilela (2010) apontam que, para reduzir a incapacidade, pode-se intervir de duas maneiras: incrementando a capacidade das pessoas segundo o modelo biomédico mais frequentemente utilizado ou ajustando a demanda das tarefas segundo os pressupostos do modelo social da deficiência e da análise da atividade.

Nas pesquisas levantadas, os pressupostos do modelo biomédico, com ações focadas no indivíduo, predominaram principalmente na literatura científica internacional. Dessa forma, observa-se que, apesar da literatura apontar para um novo modelo de incapacidade, o modo de intervir sobre a incapacidade ainda não avançou para um modelo que considere o ambiente de trabalho como participante do processo de incapacidade.

Entre as abordagens utilizadas para nortear as ações em reabilitação profissional, são citadas pelos autores: trabalho apoiado (supported work) (Bond et al., 2007; Hanrahan, Heiser, Cooper, Oulvey \& Luchins, 2006; Trotter, Minkoff, Harrison \& Hoops, 1988; Watzke \& Galvao, 2008), os programas centrados na hospitalização, sheltered workshops e reabilitação psicossocial (Watzke \& Galvao, 2008). Foram incluídas tanto abordagens que lidam com sujeitos em situação de retorno ao trabalho após afastamento como com sujeitos que estão fora do mercado de trabalho e desempregados de longa duração portadores de incapacidades.

a) Trabalho apoiado (supported employment): envolve a recolocação de pessoas com transtornos mentais severos no mercado de trabalho em conjunto com um apoio intensivo da equipe de saúde mental (Hanrahan et al., 2006). Respeitam-se as escolhas e a capacidade dos pacientes, que são colocados em empregos competitivos tão logo entram no programa. É considerada uma abordagem menos tradicional em reabilitação profissional. Os trabalhadores recebem apoio continuado por tempo indefinido para manter sua posição. É considerada a abordagem mais promissora em reabilitação profissional (Watzke \& Galvao, 2008) e citada por vários autores. Foi observado em pesquisas que a participação nos programas de apoio ao emprego é seguida de um aumento na habilidade de encontrar e manter um emprego.

b) Programas centrados na hospitalização (hospital centered programs): psiquiatras, psicólogos e trabalhadores sociais são responsáveis por identificar as necessidades dos pacientes de atingir a reabilitação profissional o mais cedo possível no curso da doença. O programa tem início com a entrada do paciente no hospital. O diagnóstico da capacidade de trabalho e das necessidades individuais tem a função de planejar e subsidiar a reabilitação. Depois de um longo período de desemprego, a terapia no hospital pode inicialmente promover o fortalecimento de algumas habilidades ocupacionais básicas (Watzker \& Galvao, 2008).

c) Oficinas protegidas (sheltered workshops): oferecem oportunidades para pacientes com as mais diversas incapacidades. Fornecem treinamento pré-profissional e, em muitos casos, a pessoa com incapacidade chega ao final da reabilitação. Os pacientes têm responsabilidades por manter o lar, preparar refeições e trabalhar na administração do lar. Depois de um período de preparação para o trabalho, são definidos locais para recolocação profissional em uma variedade de empregos temporários (Drebing, Hebert, Mueller, Ormer \& Herz, 2006; Watzker \& Galvao, 2008).

d) Reabilitação psicossocial (psychosocial rehabilitation): programas que compreendem serviços psiquiátricos bem como psicoterápicos e terapia ocupacional. Em conjunto com o processo de reabilitação, são desenvolvidas metas realísticas com o paciente a respeito do seu futuro social e profissional. Técnicas breves e focadas são aplicadas para ensinar os pacientes a encontrar um emprego no mercado de trabalho, preencher fichas, e como se comportar em entrevistas de emprego. Atividades de lazer complementam o programa. O tratamento medicamentoso e psicoterapêutico e suporte social são elementos que se integram ao programa (Watzker \& Galvao, 2008).

\section{Aspectos psicológicos em reabilitação profissional}

A literatura científica que discute os aspectos psicológicos em reabilitação é recente. Há a necessidade de pesquisas que embasem a intervenção psicológica, já que os estudos apontam a participação desses 
aspectos na incapacidade laboral tanto no adoecimento que gera incapacidade quanto nos fatores que decorrem da condição de afastamento do trabalho. Tais aspectos são descritos a seguir.

\section{Aspectos psicológicos associados às doenças decorrentes do trabalho}

Os estudos indicam uma variedade de danos psicológicos decorrentes do trabalho ou da própria situação de incapacidade. Entre eles, os transtornos mentais e do comportamento (OMS, 1993) têm sido estudados pelos autores interessados no tema da saúde e do trabalho. O nexo entre adoecimento por questões de saúde mental e o trabalho foi reconhecido a partir da Portaria do Ministério da Saúde, no 1.339/GM de 18 de novembro de 1999, que lista as doenças relacionadas ao trabalho (Brasil, 1999).

A partir disso, questões sobre saúde mental e trabalho têm preocupado pesquisadores, o que fez aumentar a produção científica sobre o assunto. No entanto, a produção científica sobre reabilitação desses trabalhadores após o reconhecimento do adoecimento mental decorrente do trabalho ainda é incipiente.

O número de trabalhadores com diagnóstico de TMC decorrentes do trabalho demonstra a necessidade de se produzirem referenciais para a atuação dos profissionais após o diagnóstico e o reconhecimento da incapacidade laboral. Dados da Previdência Social (Brasil, 2008) apresentam que entre 2006 e 2008 foram levantados 21314 casos de acidentes de trabalho dentro do Grupo F do Código Internacional de Doenças (CID), em que se encontram os casos de agravo à saúde mental. Entre os diagnósticos frequentes estão o estresse, o transtorno ansioso, o transtorno bipolar, o transtorno depressivo e o transtorno devido ao uso de álcool.

Os transtornos mentais e do comportamento têm sido destacados entre os principais motivadores de afastamento do trabalho por adoecimento. Em estudo com servidores públicos no estado de Santa Catarina, Campos e Cruz (2007) observaram que o adoecimento psicológico foi a primeira causa de afastamento do trabalho entre 2001 e 2005, seguido pelas doenças do sistema osteomolecular, do tecido conjuntivo (grupo M da CID) e do aparelho circulatório (grupo
I da (ID). Os autores chamam a atenção para a dificuldade de reabilitação desses pacientes. Em alguns casos, o agravamento da doença pode levar à aposentadoria por invalidez, o que gera consequências negativas para o trabalhador que tem seu potencial laborativo reduzido e para o sistema previdenciário devido à elevação dos custos com pagamento de benefícios.

Dados similares são encontrados em outros estudos realizados no Brasil. Siano, L. C. Ribeiro, Santiago e M. S. Ribeiro (2008), ao investigarem as perícias médicas do Instituto Nacional do Seguro Social (INSS) em Juiz de Fora, observaram que os transtornos mentais foram a terceira razão de incapacidade presumida no período de julho de 2004 a dezembro de 2006. Em Porto Alegre (RS), os TMC foram observados como diagnósticos mais frequentes entre os trabalhadores que receberam benefício no ano de 1998, juntamente com as doenças osteomoleculares e as cardiovasculares (Boff, Leite \& Azambuja, 2002). S. Sardá, Ruiz e Kirtsching (2009) apontaram a elevada prevalência de transtornos mentais em jovens trabalhadores de indústrias frigoríficas. Outro estudo aponta os TMC como o quarto grupo de diagnósticos mais frequente entre os usuários do serviço de reabilitação profissional de uma universidade pública de Minas Gerais (Sampaio, Silveira, Viana, Oliveira \& Frade, 2005).

No âmbito internacional, pesquisas mostram que um número relevante de pessoas abandona seus empregos em decorrência de TMC. Juntamente com os problemas musculoesqueléticos, o adoecimento psicológico é responsável por uma significativa parte dos afastamentos por causas médicas (Houtmann \& Kompler, 1998). Na Holanda, aproximadamente um terço das pessoas que recebem benefícios por incapacidade o fazem devido a problemas de saúde mental, a maior parte por transtornos menores que incluem o estresse. Quanto mais longos os períodos de afastamento, menor a probabilidade de retorno ao trabalho, o que resulta em uma população com uma situação financeira enfraquecida, com isolamento social e excluída do mercado de trabalho (Browers, Terluin, Tiemens \& Verhaak, 2009).

Roelen, Koopmans, Anema e van der Beek (2010) estudaram o afastamento do trabalho em um empresa pública da Alemanha no período de 2001 a 2007. A pesquisa constatou que os transtornos mentais são a 
segunda causa de afastamento, atrás somente dos distúrbios muscoloesqueléticos. Esses dois grupos de diagnóstico foram responsáveis por 58\% dos afastamentos por adoecimento, o que representa um alto encargo econômico e social, já que esses diagnósticos foram mais comuns em trabalhadores pouco qualificados e com pouco tempo de emprego. A falta de qualificação e a pouca experiência na atividade dificultam a reinserção laboral. O afastamento precoce do trabalho traz consequências tanto para o trabalhador, que se vê precocemente com dificuldades para o retorno ao mercado de trabalho, quanto para o seguro social, que tem um contingente cada vez maior de concessão de benefícios.

Nos Estado Unidos, Brodhead, Blazer, George e Tse (1990) estudaram a relação entre a depressão e os sintomas depressivos com a incapacidade e o absenteísmo no trabalho. Observaram que indivíduos com diagnóstico de transtorno depressivo têm maiores riscos de desenvolver incapacidade laboral. Outro estudo americano relaciona a depressão e a capacidade para o trabalho, e enfatiza que o comprometimento social, cognitivo e físico produzido pelo adoecimento gera perdas financeiras para o país, e resulta em acidentes de trabalho, incapacidade, dias de trabalho perdidos e produtividade reduzida (Elinson, Houck, Marcus \& Pincus, 2004).

Embora os TMC sejam apontados pelas pesquisas como os diagnósticos mais comuns, cabe lembrar que, em grande parte, eles não são notificados ou não aparecem como diagnóstico principal, já que podem estar associados a outros tipos de adoecimento, como as doenças osteomoleculares (Sardá Jr, Kupek, Cruz, Bartilotti \& Cherem, 2009), o que pode aumentar a incidência de TMC que não constam nos dados oficiais. Trabalhadores acometidos de Lesão por Esforço Repetitivo (LER) e Distúrbio Ostemuscular Relacionado ao Trabalho (DORT) geralmente apresentam sofrimento psicológico importante (Sato, Lacaz \& Bernardo, 2006). Dessa forma, é necessário atentar para esses diagnósticos, já que, em grande parte dos casos, o trabalho de reabilitação nessa área requer intervenção psicológica devido aos aspectos psicológicos relacionados.

Os aspectos psicológicos têm impacto significativo na atividade funcional de pessoas com dor crônica. Geisser, Robinson, Miller e Bade (2003) citam a ansiedade e o medo relacionados à dor, os sentimentos catastróficos e as variáveis cognitivas como crenças, pensamentos, valores, autoeficácia e estratégias de enfrentamento como aspectos relacionados à incapacidade. A autoeficácia foi apontada como um forte preditor da performance física em pacientes com dor crônica. Além disso, estão associados à dor crônica aspectos psicopatológicos como a depressão e o estresse pós-traumático. Os autores apontam que o Transtorno Depressivo Maior é observado em 30\% a 54\% das pessoas com dor crônica. Fatores relacionados à organização do trabalho, como insatisfação e estresse laboral, também aparecem como risco para o desenvolvimento de incapacidade.

Os aspectos psicológicos no contexto da reabilitação e da perda de capacidades físicas em decorrência de acidentes foram estudados por Galhordas e Lima (2004). Segundo os autores, quando ocorre uma lesão corporal, elas geralmente vêm acompanhadas de alterações da imagem corporal às quais estão relacionados sentimentos de desvalorização da imagem estética e tristeza. São comuns manifestações como ansiedade, tristeza, raiva, sentimentos de frustração, agitação, autoacusação, desespero e situações de negação de perda da incapacidade. Podem surgir ainda sentimentos de culpa, agressividade e depressão. É importante, ao avaliar os aspectos psicológicos, distinguir a patologia depressiva dos sentimentos que são comuns ao indivíduo que lida com uma perda corporal.

Dessa forma, é essencial que a intervenção tenha como objetivo auxiliar o indivíduo na tomada de consciência da lesão ou da doença, recuperar o investimento em si próprio, construindo um projeto de vida coerente com a realidade de suas capacidades após o adoecimento. Para tanto, é necessário que os profissionais de reabilitação atuem não somente sobre um corpo atingido, mas sobre os aspectos psicológicos desse indivíduo.

\section{Aspectos psicológicos associados à condição de afastamento}

O afastamento do trabalho aparece na literatura como um fator importante que pode gerar sofrimento psicológico e agravar a condição de incapacidade. 0 
sofrimento psicológico associado à condição de estar afastado e incapacitado parcialmente para o trabalho foi observado por pesquisadores (Hoefel, Jacques, Amazarray, Mendes \& Netz, 2004; Matsuo, 2002; Ramos et al., 2008; Rosin-Pinola, Silva \& Garbulho, 2004; Sardá Jr, et al. 2009; Sato et al., 2006; Takahashi \& Canesqui, 2003). A situação de afastamento do trabalho pode gerar sofrimento e desestabilizar os trabalhadores, já que o trabalho é um fator central na vida do indivíduo e no estabelecimento de modos de viver (Ramos et al., 2008).

Em pesquisa avaliativa sobre um programa de reabilitação profissional na cidade de Campinas (SP), Takahashil e Canesqui (2003) chamam atenção para o fato de trabalhadores muito jovens encontrarem-se afastados do trabalho. Segundo a autora, a ruptura nos projetos de vida representa uma perda muito significativa da identidade pessoal e profissional, tornando necessário que a intervenção o auxilie a buscar novos referenciais para que se reconheça e se construa um novo projeto de futuro.

O sofrimento psicológico decorre das situações com que o trabalhador considerado incapaz precisa lidar após o afastamento: "a perda do emprego, a não recolocação no mercado de trabalho, a recolocação em atividades com menor prestígio social, a quebra do cotidiano e as mudanças nas relações familiares... que interferem na construção da identidade profissional dos acidentados" (Rosin-Pinola et al., 2004, p.56).

Além disso, esses indivíduos ainda se deparam com situações de estigma (Matsuo, 2002) e discriminação no trabalho, na família e nos serviços de saúde (Hoefel et al., 2004). A intervenção em reabilitação profissional deve ainda lidar com a resistência do trabalhador em aceitar a limitação física e com o seu medo de voltar ao trabalho e sofrer outro acidente (Matsuo, 2002).

As consequências psicológicas podem atingir o trabalhador também quando ele retorna ao trabalho após a conclusão do processo de reabilitação profissional. Para Matsuo (2002), a mudança para uma função menos ou não qualificada possibilita pouco reconhecimento social e desenvolvimento profissional. Dessa forma, a perda do status e a impossibilidade de promoção passam a ser aspectos importantes no processo de reabilitação profissional do acidentado, que relata sentimentos de que o serviço que lhe é destinado pode ser feito por qualquer pessoa. O trabalhador acaba realizando uma atividade percebida como vazia, insignificante, desprezível, o que afeta diretamente sua autoestima e sua identidade.

Ramos et al. (2008), em relato sobre a experiência de afastamento de trabalhadores adoecidos em um hospital público de Porto Alegre, referem aspectos psicológicos decorrentes da situação de afastamento independente do diagnóstico estabelecido. Sentimentos de falha, percepção de inferioridade em relação aos pares, medo de rejeição e exclusão, sofrimento gerado pelo afastamento do trabalho, sentimento de inutilidade, acusação de simulação e sentimento de discriminação foram citados pelos trabalhadores.

Dessa forma, apesar de nem todos os trabalhadores terem diagnóstico de TMC, o sofrimento psicológico pode estar associado à situação de estar incapacitado para o trabalho. Cabe lembrar que, quanto maior o tempo de afastamento, maiores são os níveis de incapacidade e menores as possibilidade de retorno ao trabalho (Sardá Jr et al., 2009). Assim, a intervenção psicológica complementar às intervenções físicas em reabilitação profissional é importante em qualquer situação de incapacidade para diminuir o tempo de afastamento e aumentar a probabilidade de retorno ao trabalho.

Sobre a intervenção em reabilitação profissional, foram mapeadas as experiências descritas na literatura científica. Como critério de inclusão, adotou-se a intervenção psicológica em situações de afastamento do trabalho, incluídas ou não dentro de um programa estruturado ou nomeado de reabilitação profissional. Foram identificadas sete descrições de experiência em intervenção psicológica (Bartilotti et al., 2009; Hatinen et al., 2007; Lima et al., 2010; Marques et al., 2006), sendo cinco no Brasil e um na Finlândia.

Embora o número de experiências descritas nos artigos levantados seja reduzido, todas as intervenções relatadas apresentam resultados positivos que demonstram a eficácia das ações de intervenção psicológica na inclusão de trabalhadores em reabilitação. Entre os resultados apresentados, pode-se destacar o resgate da autonomia, o aumento da autoestima, a capacidade de ressignificar o futuro, o reconhecimento de estratégias de enfrentamento do adoecimento, o aumento da percepção de controle sobre o trabalho e a redução de exaustão. 


\section{Conclusão}

Os estudos no campo da reabilitação profissional e saúde mental são recentes, e observou-se aumento nas publicações na área a partir da década de 2000. Dos artigos rastreados, $65 \%$ foram publicados nesse período.

Emboa as pesquisas apontem o diagnóstico do grupo F do Código Internacional de Doenças (OMS, 1993) - Transtornos mentais e do comportamento -, entre as principais causas de afastamento do trabalho tanto no Brasil quanto nos estudos internacionais, ainda se observa uma maior atenção aos estudos com trabalhadores acometidos por problemas físicos, como as Ler/DORT. Aponta-se a necessidade da comunidade científica realizar mais estudos nessa área, ampliando a produção sobre os aspectos da saúde mental em reabilitação profissional.

Entre os principais aspectos psicológicos associados à condição de afastamento, os autores citam ruptura nos projetos de vida, perda da identidade pessoal e profissional, dificuldade de recolocação no mercado de trabalho, recolocação em atividades de menor prestígio social e menos qualificadas, quebra do cotidiano, mudanças nas relações familiares, e sentimentos de falha, de inferioridade e de inutilidade, além do preconceito gerado pela acusação de simulação. Dessa forma, a intervenção psicológica é necessária não somente em casos de diagnósticos na área de saúde mental. O suporte psicológico para trabalhadores em condição de afastamento se mostra importante para a eficácia dos programas de reabilitação profissional em geral.

Apesar do número reduzido, as experiências em reabilitação profissional na área de saúde mental descritas apresentam resultados que demonstram sua eficácia. Evidencia-se a necessidade de publicação de outras experiências de atuação em saúde mental no contexto da reabilitação profissional a fim de contribuir com a construção de modelos de intervenção na área.

Sugere-se a realização de estudos que sirvam como base para a construção de diretrizes para a atuação em saúde mental na reabilitação profissional, principalmente no campo da intervenção psicológica.

\section{Referências}

Bartilotti, C., Andrade, P. R., Varandas, J. M., Ferreira, P. C. G., \& Cabral, C. (2009). Programa de Reabilitação Ampliada (PRA): uma abordagem multidimensional do processo de reabilitação profissional. Acta Fisiatra, 16(2), 66-75.

Boff, B. M., Leite, D. F., \& Azambuja, M. I. R. (2002). Morbidade subjacente à concessão de benefício por incapacidade temporária para o trabalho. Revista de Saúde Pública, 36(3), 337-342.

Bond, G. R., Salyers, M. P., Dincin, J., Drake, R. E., Becker, D. R., Fraser, V. V., et al. (2007). A randomized controlled trial comparing two vocational models for persons with severe mental illness. Journal of Consulting and Clinical Psychology, 75(8), 968-982.

Brasil. Ministério da Previdência e Assistência Social (1999, 7 de maio). Decreto n 3.048, de 6 de maio de 1999. Aprova o regulamento da Previdência Social, e dá outras providências. Brasília: Diário Oficial da União, Seção 1, p.50.

Brasil. Ministério da Saúde. (1999). Portaria Federal no 1.339/ GM, de 18 de novembro de 1999. Institui a lista de doenças relacionadas ao trabalho, a ser adotada como referência dos agravos originadas no processo de trabalho no sistema Único de Saúde, para uso Clinico e epidemiológico. Brasília: Ministério da Saúde.

Brasil. Ministério do Trabalho e Emprego (2008). Anuário Estatístico dos Acidentes do Trabalho: AEAT. Brasília: Ministério do Trabalho e Emprego.

Browers, E. P. M., Terluin, B., Tiemens, B. G., \& Verhaak, P. F. M. (2009). Predicting return to work in employees sick-listed due to minor mental disorders. Journal of Occupational Rehabilitation, 19(4), 323-332.

Broadhead, W. E., Blazer, D. G., George, L. K., \&Tse, C. K. (1990). Depression, disability days, and days lost from work in a prospective epidemiologic survey. JAMA, 264(19), 2524-2528.

Campos, I. C. M., \& Cruz, R. M. (2007). Diagnóstico de transtornos mentais e comportamentais e relação com o trabalho de servidores públicos estaduais. In Secretaria do Estado da Administração. I Coletânea de trabalhos científicos produzidos pelos servidores públicos. Florianópolis: Secretaria do Estado da Administração.

Drebing, C. E., Hebert, M., Mueller, L. N., Ormer, E. A. V., \&Herz, L. (2006). Vocational rehabilitation from a behavioral economic perspective. Psychological Services, 3(3), 181-194.

Elinson, L., Houck, P., Marcus, S. C., \& Pincus, H. A. (2004). Depression and the ability to work. Psychiatric Services, 55(1), 29-34.

Galhordas, J. G., \& Lima, P. A. T. (2004). Aspectos psicológicos na reabilitação. Re(habilitar), 0, 35-47.

Geisser, M. E., Robinson, M. E., Miller, Q. L., \& Bade, S. M. (2003). Psychossocial factors and functional capacity evalution among persons with chronic pain. Journal of Occupational Rehabilitation, 13(4), 259-276.

Hanrahan, P., Heiser, W., Cooper, A. E., Oulvey, G., \& Luchins, D. J. (2006). Limitations of system integration in providing employment services for persons with mental illness. Administration and Policy in Mental Health and Mental Health Services Research, 33(2), 244-252. 
Hatinen, M., Kinnunen, U., Pekkonen, M., \& Kalimo, R. (2007). Comparing two burnout interventions: Perceived job control mediates decreases in burnout. International Journal of Stress Management, 14(3), 227-248.

Hoefel, M. G., Jacques, M. G., Amazarray, M. R., Mendes, J. M. R., \& Netz, J. A. (2004). Uma proposta em saúde do trabalhador com portadores de LER/DORT: grupos de ação solidária. Cadernos de Psicologia Social e do Trabalho, 7,31-39.

Houtmann, I. L. D., \& Kompler, M. A. J. (1998). Trabajo y salud mental. In Enciclopedia de Salud y Seguridad en el Trabajo (Vol.5, pp.5.1-5.23), Geneva: International Labour Organization.

International Labour Organization. (1993). Convention concerning vocational rehabilitation and employment (disabled persons). Geneva: Author. Retrieved January 20, 2009, from <http://www.ilo.org/ilolex/cgi-lex/convede. pl?C159>.

Lima, M. A. G., Andrade, A. G. M., Bulcão, C. M. A., Mota, E. M. C. L., Magalhães, F. B., Carvalho, R. C. P., et al. (2010). Programa de reabilitação profissional de trabalhadores com LER/DORT do Cesat/Bahia: ativador de mudanças na saúde do trabalhador. Revista Brasileira de Saúde Ocupacional, 35(121), 112-121.

Lollar, D. (2008). Rehabilitation psychology and public health: Commonalities, barriers and bridges. Rehabilitation Psychology, 53(2), 122-127.

Marques, A. J., Queirós, C., \&Rocha, N. B. (2006). Metodologias de reabilitação cognitiva num programa de desenvolvimento pessoal de indivíduos com doença mental e desempregados de longa duração. Psicologia, Saúde \& Doenças, 7(1), 109-116.

Matsuo, M. (2002). Acidentado do trabalho: reabilitação ou exclusão? Brasília: Ministério do Trabalho e Emprego.

Momm, W., \& Geiecker, O. (1998). Discapacidad: conceptos y definiciones. In Discapacidad y trabajo. Enciclopédia de salud y seguridad en el trabajo (Vol.17, pp.17.2-17.9). Geneva: Organización Internacional del Tabajo.

Organização Mundial de Saúde. (1993). Classificação de transtornos mentais e de comportamento da CID-10. Descrições clínicas e diretrizes diagnósticas. Porto Alegre: Artes Médicas.

Organização Mundial de Saúde. (2004). Classificação internacional de funcionalidade, incapacidade e saúde. Lisboa: Autor.

Pledger, C. (2003). Discourse on disability and rehabilitation issues: Opportunities for psychology. American Psychologist, 58(4), 279-284.

Ramos, M. Z., Tittoni, J., \& Nardi, H. C. (2008). A experiência de afastamento do trabalho por adoecimento vivenciada como processo de ruptura ou continuidade nos modos de viver. Cadernos de Psicologia Social e do Trabalho, 11 (2), 209-221.
Reagles, K. W., Wright, G. N., \& Butler, A (1971). Rehabilitation gain: Relationship with client characteristics and counselor intervention. Journal of Counselling Psychology, 18(5), 490-495.

Roelen, C. A. M., Koopmans, P. C., Anema, J. R., \& van der Beek, A. J. (2010). Recurrence of medically certified sickness according to diagnosis: A sickness absence register study. Journal of Occupational Rehabilitation, 20(1), 113-121.

Rosin-Pinola, A. R., Silva, C. P., \& Garbulho, N. F. (2004). Implicações psicossociais para o acidentado reinserido no mercado de trabalho e desempregado. Revista Brasileira de Orientação Profissional, 5(2), 53-62.

Sampaio, R. F., Silveira, A. M., Viana, S. O., Oliveira, G. B. A., \& Frade, F. (2005). Implantação do serviço de reabilitação profissional:a experiência da UFMG. Fisioterapiae Pesquisa, 12(2), 28-34

Sardá, S., Ruiz, R., \& Kirstching, G. (2009). Tutela jurídica da saúde dos empregados de frigoríficos: considerações dos serviços públicos. Acta Fisiatrica, 16(2), 59-65.

Sardá Jr., J. J., Kupek, E., Cruz, R. M., Bartilotti, C., \& Cherem, A. J. (2009). Preditores de retorno ao trabalho em uma população de trabalhadores atendidos em um programa de reabilitação profissional. Acta Fisiatra, 16(2), 81-86.

Sato, L., Lacaz, F. A., \& Bernardo, M. H. (2006). Psicologia e saúde do trabalhador: práticas e investigações na saúde pública de São Paulo. Estudos de Psicologia (Natal), 11(3), 281-288.

Siano, A. K., Ribeiro, L. C., Santiago, A. E., \& Ribeiro, M. S. (2008). Relevância dos transtornos mentais entre as perícias médicas requerentes de auxílio-doença na gerência executiva do Instituto Nacional de Seguro Social de Juiz de Fora - Minas Gerais. HU Revista, 34(4), 235-242.

Simonelli, A. P., Camarotto, J. A., Bravo, E. S., \& Vilela, R. A. G. (2010). Proposta de articulação entre abordagens metodológicas para melhoria do processo de reabilitação profissional. Revista Brasileira de Saúde Ocupacional, 35(121), 64-73.

Takahashil, M. A. B. C., \& Canesqui, A. M. (2003). Pesquisa avaliativa em reabilitação profissional: a efetividade de um serviço em desconstrução. Cadernos de Saúde Pública, 9(5), 1473-1483.

Trotter, S., Minkoof, K., Harrison, K., \& Hoops, J. (1988). Supported work: An innovative approach to the vocational rehabilitation of persons who are psychiatrically disabled. Rehabilitation Psychology, 33(1), 27-36.

Watzke, S., \& Galvao, A. (2008). The feasibility of vocational rehabilitation in subjects with severe mental illness. Salud Pública de México, 50(2), S260-S262.

Recebido em: 26/7/2011

Versão final em: 4/4/2012

Aprovado em: 21/5/2012 
